\title{
Update on Genetic Testing and Warfarin
}

\author{
Snehal H. Bhatt
}

Published online: 27 June 2014

(c) Springer Science+Business Media New York 2014

\begin{abstract}
Warfarin therapy is complex and often requires careful consideration of multiple patient specific factors during initiation and chronic management. Identification of genetic variants that influence warfarin response via pharmacogenetic testing provides an opportunity to enhance the quality of therapy. Many studies have attempted to identify how genetic testing can improve warfarin management. Due to the variability in the study results and the lack of clinical outcome data, the true benefits of pharmacogenetic testing for warfarin remain unclear. If genetic testing has been obtained or is readily available prior to warfarin initiation, it is reasonable to incorporate genetic variants into the decision making process for the initial and suspected maintenance warfarin dose. If genetic testing is not readily available, initiating and managing warfarin using clinical characteristics alone compares similarly to pharmacogenetic based algorithm. The true impact of genetic testing on clinical outcomes requires further investigation.
\end{abstract}

Keywords Warfarin - Genetics · Genotype · Dosing · Algorithm $\cdot$ Pharmacogenetics

\section{Introduction}

Warfarin is a vitamin-K antagonist (VKA) oral anticoagulant that has been used for the management of thromboembolic events for over 50 years. Warfarin exerts its anticoagulant effect by inhibiting the vitamin-k epoxide reductase complex subunit 1 (VKORC1), which results in a

S. H. Bhatt $(\square)$

MCPHS University, 179 Longwood Avenue, Boston, MA 02115, USA

e-mail: sbhatt@bidmc.harvard.edu reduction in the synthesis of the hepatic coagulation factors II, VII, IX, and X [1•]. Despite this vast clinical experience, initiating and maintaining warfarin therapy remains complex, and the optimal approach for initiating therapy remains unknown. Warfarin dosing can vary up to 10 -fold among patients, and other factors, such as age, weight, ethnicity, organ function, diet, and concomitant medications necessitate the need to individualize therapy $[2 \bullet, 3]$. Because potentially disastrous effects can occur (i.e., thrombosis or major bleeding) when warfarin dosing and response results in anticoagulant effects outside the therapeutic range (commonly defined as international normalized ratio (INR) of 2-3), strategies to optimize warfarin therapy are necessary.

Recently, pharmacogenetic information has gained interest as a means to further individualize drug therapy, and warfarin has been considered a prototypical medication for the potential for improving patient care by obtaining pharmacogenetic information. Variations in two genes may account for some of the significant variability observed in determining warfarin response: VKORC1, the pharmacologic target for warfarin, and cytochrome P450, family 2, subfamily C, polypeptide 9 (CYP2C9), the main enzyme involved in warfarin metabolism. Two relatively common variations in the genes encoding $\mathrm{CYP} 2 \mathrm{C} 9$, referred to as $C Y P 2 C 9 * 2$ and $C Y P 2 C 9 * 3$, have been found to alter warfarin metabolism. Patients with $C Y P 2 C 9 * 2$ variants have a $30 \%$ reduction in CYP 2C9 activity, whereas patients with $C Y P 2 C 9 * 3$ have an $80 \%$ reduction in warfarin metabolism, resulting in elevated warfarin concentrations in patients, which could result in an increased risk for major bleeding [4].

Mutations in VKORC1 have been associated with a deficiency in vitamin-K-dependent clotting factors, resulting in altered warfarin response. Specifically, a common 
noncoding variant $(-1639 \mathrm{G}>\mathrm{A}$, rs9923231) has been associated with a significant reduction in warfarin dosing requirements secondary to increased warfarin sensitivity $[5,6]$. Based on these data, and for the promise that early identification of genetic information could improve warfarin therapy [7-14], the Food and Drug Administration (FDA) has twice revised the prescribing information for warfarin. The first revision was intended to increase awareness that genetic testing for VKORC1 and CYP2C9 could be considered when initiating warfarin therapy if available, while the second revision provided a table of dosing considerations and expected maintenance doses for patients with either singular or combination genotypic variations within the prescribing information [15]. Presently, the FDA does not recommend routine genetic testing for warfarin therapy, and the Centers of Medicare and Medicaid Services do not cover the costs of genotype testing when initiating warfarin therapy [16].

The initial enthusiasm for genetic testing resulted in many evaluations of the impact of warfarin genetic testing. While initially promising, the results of these studies were mixed and were limited by either lack of randomization, small sample size, diverse patient population, and most importantly, lack of ability to measure clinical outcome endpoints [8]. Most of these studies focused on the development of dosing algorithms and the improvement of those algorithms with the addition of genetic information, the ability of genotype guided dosing algorithms to predict stable maintenance warfarin doses when compared to either clinical algorithms alone, or fixed-dose algorithms.

One of the more promising pharmacogenetic warfarin dosing algorithms was recently evaluated in a study that was actually a combination of two different prospective clinical comparisons within a single, integrated healthcare system consisting of three hospitals [17•]. The first component was a blinded, randomized comparison of two refined genotypeguided warfarin dosing algorithms, whereas the second objective was to compare the clinical effectiveness of either of the pharmacogenetic algorithms with a parallel, standard, but non-pharmacogenetic information based dosing strategy. The two different pharmacogenetic algorithms were classified as either a one-step or a three-step algorithm pharmacogenetic algorithm. Major differences between these two algorithms are as follows. The one- step algorithm was based on the published International Warfarin Pharmacogenetics Consortium (IWPC) dosing algorithm [18•], which was derived from a cohort of almost 5,000 patients. The three-step algorithm included two modifications from the IWPC based algorithm. First, the three-step algorithm did not take into account CYP2C9 variant status for the first two days of initiating therapy, and this was replaced with a special dose-revision algorithm as generated by the International Dose-Revision Collaborators [19]. This dosing revision was considered on the patients' day 4 (or day 5) INR, provided that the patient had received 3 or 4 warfarin doses. Both algorithms incorporated clinical information as part of the decision making: sex, age, height, weight, smoking status, race/ethnicity, target INR, pertinent medications known to interact with warfarin, and the option for administering a warfarin loading dose. In the pharmacogenetic dosing groups, INRs were obtained at baseline, on day 4 or 5 ; on days $8,14,21$, and 30 ; then, monthly thereafter. Additional INRs were allowed at clinician discretion as needed in order to achieve and maintain stable therapeutic INRs.

Patients enrolled in the standard dosing arm were identified by a query of electronic medical records databases within the participating hospitals. These patients all received warfarin therapy at the same time as the pharmacogenetic groups, but the dosing algorithm used was a standard, fixed dose regimen that did not consider pharmacogenetic information. It was generally assumed that patients in the standard dosing arm would receive a fixed $5 \mathrm{mg}$ initial warfarin dose, with subsequent doses left to the discretion of the individual physician or healthcare provider within the three hospitals. Subsequent dosing modifications were based on INR response, in accordance with promoted and generally recommended healthcare systems guidelines used within the three hospitals.

A total of 504 patients were enrolled in the two pharmacogenetic guided algorithm arms, and these patients were followed for three months, unless warfarin therapy was clinical indicated for $<3$ months of total duration. The primary objective of this study was to determine if pharmacogenetic guided dosing strategies resulted in an increase in the percentage of time patients were within the warfarin therapeutic range (INR 2-3). Compared to the 1,866 patients in the standard dosing arm, patients in either of the pharmacogenetic dosing arms experienced a significantly greater time spent within the therapeutic range at both 30 days and 3 months (68.9 versus $58.4 \%$ at 30 days, $P<0.001 ; 71.2$ versus $58.6 \%$ at 3 months; $P<0.001$ ). While these results appear to support the use of pharmacogenetic guided warfarin dosing, it should be noted that the differences observed could be due to the more frequent INR monitoring specified in the pharmacogenetic arms, and that the dosing strategy in these arms accounted for greater clinical criteria overall. The study was also not powered to detect clinical outcome differences between groups.

Recently, published studies have attempted to further clarify the role of pharmacogenetic testing. The first trial was the Clarification of Optimal Anticoagulation through Genetics (COAG) trial, which was a multicenter, doubleblind, randomized controlled trial that compared a genotype guided warfarin dosing strategy with a clinically based dosing strategy during the first five days of warfarin therapy [20••]. Adult patients aged 18 years or older who were 
starting warfarin therapy with a target INR of $2-3$ were randomly assigned to either the clinical or pharmacogenetic warfarin dosing strategy, and randomization was stratified according to self-reported ethnicity (blacks versus nonblack). Patients were followed for a total of six months, and the primary objective of the COAG trial was the percentage of time that the INR was in the therapeutic range from day 4-5 through day 28 of therapy. Importantly, the COAG trial attempted to evaluate clinical outcomes, but these were combined within a composite of secondary endpoints. Specifically, the secondary outcomes included a composite of: any INR value of 4 or greater, major bleeding, or thromboembolism events occurring in the first 4 weeks; the time to the first therapeutic INR; the time to maintenance dose determination; and the time to an adverse event such as death from any cause, major bleeding, thromboembolism, or any clinically relevant nonmajor bleeding event within the first 4 weeks.

A total of 1,015 patients were enrolled and randomly assigned to either the genotype-guided dosing algorithm or the clinically guided dosing algorithm. Unlike many previous studies, there was no significant difference in primary endpoint between groups $(45.2 \%$ in the genotype-guided group versus $45.4 \%$ in the clinically guided group). Interestingly, there was a difference between groups in the subset of black patients. Black patients enrolled in the clinical dosing group experience a time in therapeutic range of $43.5 \%$ compared to only $35.2 \%$ in the genetic guided group $(P=0.01)$. There were no significant differences in the principal secondary outcome (the time to any INR of $\geq 4$, major bleeding, or thromboembolism) or any other adverse events between groups.

On the heels of what appeared to be a disappointing result, the EU-PACT group simultaneously published two studies of pharmacogenetic-guided dosing strategies with warfarin, and either acenocumarol or phenprocoumon, respectively $[21 \bullet \bullet, 22]$. These two separate studies interestingly conflicted with each other and with the results observed the COAG study. The EU-PACT warfarin study was a multicenter, randomized, controlled trial involving 455 adult patients requiring warfarin therapy for either atrial fibrillation or venous thromboembolism with a target INR of 2-3. Patients assigned to the genotype-guided group, warfarin doses were prescribed according to a pharmacogenetic-based algorithm for the first 5 days, whereas patients in the control (standard dosing) group received a 3-day loading-dose regimen. Patients 75 years or older receiving $5 \mathrm{mg}$ daily for the first three days, whereas patients aged $<75$ years-old received $10 \mathrm{mg}$ on Day 1 followed by $5 \mathrm{mg}$ on days $2-3$. After these initiation periods, all patients were managed according to routine clinical practice and all patients had INR measurements obtained days $1,4,6,8,15,22,57$, and 84 . The primary outcome of the EU-PACT warfarin trial was the percentage of time in the therapeutic range of 2.0-3.0 for the INR during the first 12 weeks after warfarin initiation.

Due to slower enrollment, a minimal sample size of 200 patients per group was set as the target in order to have an $80 \%$ power to detect a $7 \%$ difference in the primary endpoint. At the end of the 12-week study period, the mean percentage of time in the therapeutic range was $67.4 \%$ in the genotype-guided group as compared with $60.3 \%$ in the control group (adjusted difference, $7.0 \%$ points; $95 \%$ confidence interval, 3.3-10.6; $P<0.001$ ). There were significantly fewer incidences of excessive anticoagulation (INR $\geq 4.0$ ) in the genotype-guided group. The median time to reach a therapeutic INR was 21 days in the genotype-guided group as compared with 29 days in the control group $(P<0.001)$. With regards to clinical outcome endpoints, there were no cases of major bleeding observed during the trial, and there were no differences in overall bleeding events between groups.

The second EU-PACT trial was conducted as two single-blind, randomized trials comparing the initiation of either acenocoumarol or phenprocoumon for the treatment of patients with either atrial fibrillation or venous thromboembolism [22]. Patients were randomized to either a genotype-guided dosing algorithm that included clinical variables and genotyping for CYP2C9 and VKORC1, or a dosing algorithm that included only clinical variables. The primary outcome was the percentage of time in the target range for the INR (INR; target range, 2.0-3.0) in the 12-week period after the initiation of therapy. Owing to low enrollment, the two trials were combined for analysis. The primary outcome was assessed in patients who remained in the trial for at least 10 weeks.

A total of 548 patients were enrolled (273 patients in the genotype-guided group and 275 in the control group). The follow-up was at least 10 weeks for 239 patients in the genotype-guided group and 245 in the control group. The percentage of time in the therapeutic INR range was $61.6 \%$ for patients receiving genotype-guided dosing and $60.2 \%$ for those receiving clinically guided dosing $(P=0.52)$. There were no significant differences between the two groups for several secondary outcomes. The percentage of time in the therapeutic range during the first 4 weeks after the initiation of treatment in the two groups was 52.8 and $47.5 \%(P=0.02)$, respectively. There were no significant differences with respect to the incidence of bleeding or thromboembolic events.

\section{Discussion}

Warfarin therapy remains complex, and the value of the identification of genetic variants as a means to improve 
warfarin therapy is still unclear. Results of recent randomized controlled trials have provided conflicting results as to the overall magnitude of benefit, and the true benefit of genetic-guided dosing on clinical outcomes of importance (major bleeding, recurrent thrombotic events) remains unknown. The recent COAG and EU-PACT studies do not discourage the use of genetic testing, but rather temper the enthusiasm for widespread adoption.

Differences observed between the COAG and EUPACT trials could be explained by differences in the pharmacogenetic algorithms used in the two trials, and because of differences in the manner in which warfarin was managed within the control groups. In the COAG trial, the control group was dosed with a very thorough clinical dosing algorithm consisting of very important patient specific factors. Therefore, the addition of genetic information on top of these important variables might have reduced the overall impact genetic testing. In fact, depending upon the number of clinical variables used in determining warfarin therapy, the addition of genetic information only slightly improves the ability to predict the proper therapeutic warfarin maintenance dose [18•].

The EU-PACT trials provided conflicting results, when compared to both the COAG trial, and to each other. While the warfarin trial showed a benefit to pharmacogenetic testing, the results were not replicated when alternative Vitamin $\mathrm{K}$ antagonists were used. With regards to possible explanations for the positive results observed in the EUPACT warfarin trial, it is possible that smaller sample size and low enrollment influenced the results observed. Additionally, the fixed dosing algorithm of 5-10 $\mathrm{mg}$ doses used in the control group increased the overall variability of warfarin response in the control group, thus reducing the initial time in therapeutic range for this group. In fact, the greatest difference in time in therapeutic range in this trial was observed soon after initiation of therapy, and became less pronounced as the trial progressed.

There are several additional points to consider that may help place these conflicting results into context clinically. The majority of the published clinical trials, including the three recent publications, required frequent INR measurement irrespective of the participant's assigned intervention group. Therefore, it is possible that even though the primary endpoints of time in therapeutic range were different, the frequent INR measurements may have prevented clinical outcomes from occurring due to dosing adjustments made at the time of each of those out-of-range visits [23]. Similarly, while an overall $7 \%$ absolute difference in timein therapeutic range may be statistically significant, the fact that the control group still experienced a time in therapeutic range of $60 \%$ suggests that the control group in the EU-PACT warfarin trial still received quality management. Thus, it is plausible that the $7 \%$ improvement in time-in therapeutic range observed in the EU-PACT warfarin genetic-guided group was not meaningful enough to truly influence differences in clinical outcomes.

Additionally, the frequency of INR monitoring mandated in the COAG and EU-PACT trials may not reflect the entire spectrum of warfarin management clinically. Depending upon the location, indication for warfarin, patient preference, and many other important factors, many warfarin-treated patients may not be managed by anticoagulation clinics or other settings where frequent monitoring is the standard of care. Even in such settings, patients may be either unable or unwilling to comply with frequent INR measurements. These common clinical scenarios were not a part of the dosing protocols in clinical trials, and it is possible that a genetic-guided dosing approach may improve the overall quality of warfarin management in these types of patients.

While the recent data do not validate the use of genetic testing to guide warfarin therapy, they do not refute the potential benefits. In centers where genetic testing has been obtained or is readily available prior to warfarin initiation, it is reasonable to incorporate genetic variants into the decision making process for the initial and suspected maintenance warfarin dose. If genetic testing is not readily available, initiating and managing warfarin using clinical characteristics alone compares similarly to pharmacogenetic based algorithm. The true impact of genetic testing on clinically important outcomes such as prevention of thrombosis or bleeding complications requires further investigation.

\section{Compliance with Ethics Guidelines}

Conflict of Interest Snehal H. Bhatt: Speakers Bureau, Janssen Pharmaceuticals, Inc.

Human and Animal Rights and Informed Consent This article does not contain any studies with human or animal subjects performed by any of the authors.

\section{References}

Recently published papers of particular interest have been highlighted as:

- Of importance

•- Of major importance

1. - Ageno W, Gallus AS, Wittkowsky A, et al. Oral anticoagulant therapy: antithrombotic therapy and prevention of thrombosis, $9^{\text {th }}$ ed: American College of Chest Physicians Evidence-Based Clinical Practice Guidelines. Chest. 2012;141(Suppl 2):e44S$88 \mathrm{~S}$. These are the most recent clinical practice guidelines, and they provide a thorough review of warfarin pharmacology, dosing strategies, INR monitoring, drug interactions and practical management guidance. 
2. - Lee CR, Goldstein JA, Piepe JA. Cytochrome P450 2C9 polymorphisms: a comprehensive review of the in vitro and human data. Pharmacogenetics. 2002;12(3):251-63. This paper provides a nice overview of CYP 2 C9 polymorphisms and its potential application to warfarin dosing.

3. Gage BF, Eby C, Johnson JA, et al. Use of pharmacogenetic and clinical factors to predict the therapeutic dose of warfarin. Clin Pharmacol Ther. 2008;84(3):326-31.

4. Higashi MK, Veenstra DL, Kondo LM, et al. Association between CYP2C9 genetic variants and anticoagulation-related outcomes during warfarin therapy. JAMA. 2002;287(13):1690-8.

5. Rieder MJ, Reiner AP, Gage BF, et al. Effect of VKORC1 haplotypes on transcriptional regulation and warfarin dose. N Engl J Med. 2005;352(22):2285-93.

6. Yin T, Miyata T. Warfarin dose and the pharmacogenomics of CYP2C9 and VKORC1 - rationale and perspectives. Thromb Res. 2007;120(1):1-10.

7. Limdi NA, Wadelius M, Cavallari L, et al. International Warfarin Pharmacogenetics Consortium. Warfarin pharmacogenetics: a single VKORC1 polymorphism is predictive of dose across 3 racial groups. Blood. 2010;115(18):3827-34.

8. Anderson JL, Horne BD, Stevens SM, et al. Randomized trial of genotype guided versus standard warfarin dosing in patients initiating oral anticoagulation. Circulation. 2007;116(22):2563-70.

9. Caraco Y, Blotnick S, Muzkat M. A CYP2C9 genotype-guided warfarin prescribing enhances the efficacy and safety of anticoagulation: a prospective, randomized, controlled study. Clin Pharmacol Ther. 2008;83(3):460-70.

10. Burmester JK, Berg RL, Yale SH, et al. A randomized controlled trial of genotype-based coumadin initiation. Genet Med. 2011;13(6):509-18.

11. Epstein RS, Moyer TP, Aubert RE, et al. Warfarin genotyping reduces hospitalization rates results from the MM-WES (MedcoMayo Warfarin Effectiveness Study). J Am Coll Cardiol. 2010;55(25):2804-12.

12. Voora D, Eby C, Linder MW, et al. Prospective dosing of warfarin based on cytochrome p450 2C9 genotype. Thromb Haemost. 2005;93:700-5.

13. Millican E, Jacobsen-Lenzini PA, Milligan PE, et al. Geneticbased dosing in orthopaedic patients beginning warfarin therapy. Blood. 2007;110(5):1511-5.

14. Lenzini PA, Grice GR, Milligan PE, et al. Laboratory and clinical outcomes of pharmacogenetic vs. clinical protocols for warfarin initiation in orthopedic patients. J Thromb Haemost. 2008;6(10): 1655-62.

15. Br istol-Myers Squibb. Coumadin (warfarin sodium) package insert. http://packageinserts.bms.com/pi/pi_coumadin.pdf. Accessed 1 Apr 2014.
16. Centers for Medicare and Medicaid Services. Medicare coverage database. Available at http://www.cms.gov/medicare-coveragedatabase/search/advanced-search.aspx. Accessed 3 Apr 2014.

17. - Anderson JL, Horne BD, Stevens SM, et al. A randomized and clinical effectiveness trial comparing two pharmacogenetic algorithms and standard of care for individualizing warfarin dosing (Coumagen II). Circulation. 2012;125(16):1997-2005. This study evaluated one of the validated pharmacogenetic dosing algorhythms versus a more complicated 3 step dosing algorhythm. The results suggest that the simplified 1 step genetic dosing approach was non-inferior to a more complex 3 step approach.

18. - The International Warfarin Pharmacogenetics Consortium. Estimation of the warfarin dose with clinical and pharmacogenetic data. N Engl J Med. 2009;360(8):753-64. This paper contains one of the two validated warfarin genetic dosing algorhythms, which was validated in a large cohort of more than 5000 patients.

19. Lenzini PA, Wadelius M, Kimmel SE, et al. Integration of genetic, clinical, and INR data to refine warfarin dosing. Clin Pharmacol Ther. 2010;87(5):572-8.

20. • Kimmel SE, French B, Kasner SE, et al. A pharmacogenetic versus a clinical algorithm for warfarin dosing. N Engl J Med. 2013;369(24):2283-93. This is the largest of the three recent publications evaluating the clinical impact of warfarin pharmacogenetic guided dosing compared with clinical dosing in a randomized, double-blind, multicenter controlled trial. There was no differences in time in therapeutic range, or clinical outcomes between groups.

21. •• Pirmohamed M, Burnside G, Eriksson N, et al. A randomized trial of genotype-guided dosing of warfarin. N Engl J Med. 2013;369(24):2294-303. This was the second of three recent publications attempting to clarify the clinical impact of warfarin pharmacogenetic guided dosing. This slightly smaller study showed that pharmacogenetic testing resulted in a greater time in therapeutic range compared to a fixed dose initial warfarin therapy regimen, although no differences in clinical outcomes were observed.

22. Verhoef TI, Ragia G, deBoer A, et al. A randomized trial of genotype-guided dosing of acenocumarol and phenprocoumon. N Engl J Med. 2013;369(24):2304-12.

23. Zineh I, Pacanowski M, Woodcock J. Pharmacogenetics and coumarin dosing-recalibrating expectations. $\mathrm{N}$ Engl $\mathrm{J}$ Med. 2013;369(24):2273-5. 